\begin{tabular}{c} 
International Journal of Engineering \& Technology, 7 (3) (2018) 1666-1671 \\
International Journal of Engineering \& Technology \\
SPC \\
Website: $\begin{array}{c}\text { ww. sciencepubco.com/index.php/IJET } \\
\text { doi: } 10.14419 / \text { ijet.v7i3.15409 } \\
\text { Research paper }\end{array}$ \\
\hline
\end{tabular}

\title{
Path-loss modelling for WSN deployment in indoor and outdoor environments for medical applications
}

\author{
Ahmed Bashar Fakhri ${ }^{1}$ *, Sadik Kamel Gharghan ${ }^{1}$, Saleem Latteef Mohammed ${ }^{1}$ \\ ${ }^{I}$ Department of Medical Instrumentation Techniques Engineering, Electrical Engineering Technical College, \\ Middle Technical University, Al Doura 10022, Baghdad-Iraq \\ *Corresponding author E-mail: ahmedbashar85@yahoo.com
}

\begin{abstract}
Wireless sensor networks (WSNs) and their applications have received significantly interested in the last few years. In WSN, knowing an accurate path-loss model as well as packet delivery should be taken into account for the successful distribution of several nodes in the network. This paper presents a path-loss modeling and performance evaluation of the ZigBee wireless standard. Received signal strength indicator (RSSI) measurements were achieved in outdoor and indoor environments to derive the path-loss based on Log-Normal Shadowing Model (LNSM). The path-loss parameters such as standard deviation and path loss exponents were estimated over point-to-point ZigBee WSN. In addition, the variances of received RSSI values and standard deviation for these values have been investigated. Furthermore, the data packets received is measured practically. Results revealed that the LNSM can be estimated to reflect the channel losses in both outdoor and indoor environments for medical application. The data delivery was achieved successfully of $100 \%$ in outdoor which better than indoor due to multipath propagation and shadowing. Moreover, the data packets delivery of the current work outperformed previous work.
\end{abstract}

Keywords: LNSM; Path Loss Model; RSSI; WSN; ZigBee.

\section{Introduction}

One of the most common research topic is wireless communication, in current daily life. Wireless sensor network (WSN) have become motivating study fields, synchronized with the production of tiny and cheap of radio modules, and microcomputers. WSN evolved of many wireless nodes deployed in a large area which have the capability to sense diverse events depended on the type of application. There are large applications that can be used for WSN: e.g., monitoring [1] and healthcare [2], sport [3], agriculture [4], disasters, etc.. Several wireless technology are used in WSN such as Bluetooth, $\mathrm{WiFi}$, and ZigBee. ZigBee wireless protocol is commonly use of in WSN because of its advantages $[5,6]$ which represented by low power consumption, inexpensive, sleep mode algorithm acceptation for saving energy, and easily to deploy with suitable battery life. The data rate speed of ZigBee is $250 \mathrm{kbps}$ [7], the latency time is 6 or $10 \mathrm{~ms}$. ZigBee has the ability for data transition between WSN nodes to control and monitor different systems [8], [9]. Three main types of XBee like S1, S2, and S2C. XBee S2C has been chosen in the current work because of low power consumption of 33 $\mathrm{mA}$ as well as communication distance (60 indoor and 1200 outdoor [10]) when compared with XBee S1 and S2. Where, S2 and S1 consume 40 and $50 \mathrm{~mA}$, respectively. In addition, both of S1 and S2 has limited communication distance, especially in indoor circumstance, where the distance up to 30 and $40 \mathrm{~m}$ for S1 and S2, respectively [11]. However, the communication distance is improved in outdoor environment for S1 and S2 relative to indoor, where the communication distance of 100 and $120 \mathrm{~m}$, respectively [12]. Several propagation characteristics have been widely studied for indoor channels which it dedicated on precise indoor environments [1317].
There are various channel propagation models are used for wireless communications that forecast received signal strength loss versus distance path loss. There are three types are commonly employed for WSN: (i) free space propagation model is based on the theory that the transmitter and receiver are in the line of view, in addition it assumes no barriers between them [18], (ii) two-Ray ground model it is like free space propagation with addition reflection. A receiver of two-ray ground model obtains direct communication ray and reflected ray[18], and (iii) log-distance model has been obtained from empirical and analytical methods [18] and it well known as Long-Normal Shadowing Model (LNSM). ZigBee modules support Received Signal Strength Indicator (RSSI) for every received data packet. The RSSI is combined with path loss and shadowing model to produce the LNSM to estimate the distance between network nodes [19]. As well as, known many channel parameters (i.e., path loss exponent and standard deviation) which can measure channel environments type. The RSSI method is has some features represented by cheap[20] which that mean it is not need additional hardware, antenna array, and time synchronization. However, methods based on RSSI has low distance estimation accuracy.

In the current paper, the LNSM type is adopted due to common use. The characteristics of signal propagation of wireless channels under indoor and outdoor in Electrical Engineering Technical College environment have been investigated. The experimental results show a new experiential path-loss model can derive based on the RSSI of ZigBee and distance between transmitter and receiver. The LNSM is derived by using linear regression for both indoor and outdoor. In addition, the statistical parameters of the environments for both models have been measured. The current work is focused for use in medical applications, where the ZigBee mobile node (i.e., transmitter) is represented a patient carrying wearable monitoring wireless 
device. Whereas, the ZigBee receiver node represents the monitoring base station in the medical staff monitoring room.

The contributions of the paper can be summarized as follow:

1) Measured the RSSI in real outdoor and indoor environments.

2) The physical parameters (i.e., the path loss exponent and standard deviation) for both indoor and outdoor surrounding are measured which reflect the quality of transmission channel.

3) Derived a path loss model for indoor and outdoor environment based on real measurements.

\section{Path loss model based on LNSM}

The signal is affected by the channel environments during transmission in wireless channel. Consequently, to estimate parameters that affect the strength of the received signal, the channel must be modelled by using a particular model. The most popular propagation model, namely [21] LNSM. LNSM is the most appropriate model for WSN uses because of its general nature and environment-based setting [22]. Many impairments will be attenuated or affected on the strength of the transmitted signal such as reflection, diffraction, and scattering. These impairments are deliberated the main three mechanisms that effect the propagation on the wireless channel. Most wireless channel models are derived on the basis of a combination of experimental and analytical methods. The experimental method is based on curve fitting, while the analytical method be depending on measured data. This has the benefit of taking into consideration the indirectly of all propagation features in the channel through the actual area measurements. While, the related research works evaluate both outdoor and indoor environments based on ZigBee wireless technology [2], [23-27], these works did not truthfully fit to our application (i.e. medical application).

Most wireless ZigBee elements support RSSI, which that means the received power can be determined for each received packet. The power of a signal moving between two nodes is the signal parameters, which include the information that reveals the distance between those nodes. These parameters can be also used for distance estimation along with LNSM and path loss.

Therefore, the LNSM can be given as [28]:

$\mathrm{WL}(\mathrm{l}) \mathrm{dBm}=\mathrm{Wo}\left(\mathrm{l}_{0}\right)+10 \beta \log 10\left(\mathrm{l} / \mathrm{l}_{0}\right)+\mathrm{x}$

where $\mathrm{WL}(\mathrm{l}) \mathrm{dBm}$ is the path loss at a distance $\mathrm{d}$ in meters, $\mathrm{W}_{\mathrm{o}}\left(\mathrm{l}_{0}\right)$ is the path loss power at a reference distance $d_{o}$ (i.e., 1 meter, which is considered in this study); it can be acquired from field measurements or by calculating using the Friis equation; $\beta$ is the path loss exponent that shows the rate at which the RSS decreases with distance, and $\sigma$ is standard deviation (in decibels). The RSSI at the movable node can be calculated from (2) as follows [29].

$$
\operatorname{RSSI}(\mathrm{dBm})=\mathrm{Wt}(\mathrm{dBm})-\mathrm{WL}(\mathrm{l}) \mathrm{dBm}
$$

Where Wt is the node transmitted power in $\mathrm{dBm}$. Then, the RSSI at the movable node will be [30-32].

$$
\operatorname{RSSI}(\mathrm{dBm})=\mathrm{Wt}(\mathrm{dBm})-\mathrm{W}_{0}-10 \beta \log 10\left(\frac{\mathrm{l}}{\mathrm{I}_{0}}\right)+\sigma
$$

Your paper must be in two column format with a space of $0.5 \mathrm{~cm}$ between columns.

\section{Experiments setup and configuration for outdoor and indoor}

ZigBee wireless protocol is a technical created for controlling and sensor networks established on the IEEE 802.15.4 standard. It can be used universally, authoritative and secure. ZigBee supports a big number of nodes about 65,000 [11]. Path loss model which consist of the path loss exponent and standard deviation parameters can be determined based on WSN using two ZigBee. First one as a transmitter node and the second one as the receiver node. The experiment was executed in Electrical Engineering Technical College for both outdoor and indoor environments. Both of transmitter and receiver nodes are in over the surface of the ground about $1.5 \mathrm{~m}$ to prevent interference caused by Fresnel zone. The transmitter node is fixed in a specific position in the tested area, whereas the receiver is moving away from the transmitter in pre-planning positions. The data packets were received by the receiver node from the transmitter node. The receiver node receives the data packets of the transmitter node and the RSSI values will be recorded by X-CTU software program in the laptop. The X-CTU software program is used to configure and control ZigBee module (i.e., XBee S2) [33]. The transmitted power of ZigBee is set at 4 level (i.e., $5 \mathrm{dBm}$ ) [10]. The Setup configuration of ZigBee transmitter node and receiver node was shown in Figure 1. The adopted WSN is consist of two nodes, (i) transmitter node which it involves of battery $(3.7 \mathrm{~V}, 1300 \mathrm{mAh})$ and XBee (SC2). (ii) Receiver node which consists of XBee (Series 2) connected to the laptop to measure the RSSI values. The RSSI value can be used to estimate the path loss model with its physical parameters. The path loss model is measured in two environments outdoor and indoor based on two experiments as explained in the next section.

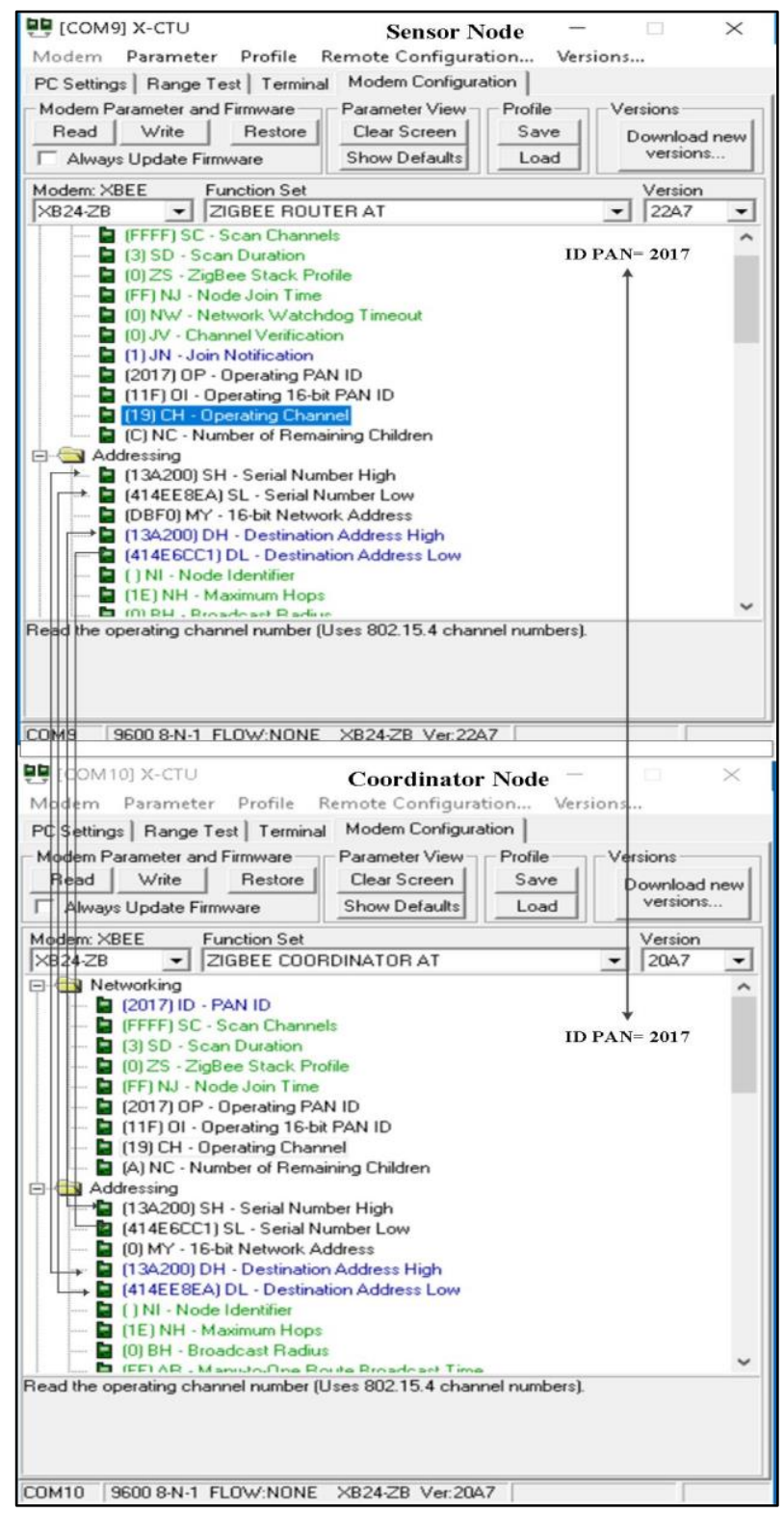

Fig. 1: Setup Configuration of Zigbee Transmitter Node and Receiver Nodes. 
The outdoor experiment was conducted in car parking of Electrical Engineering Technical College, where it has the area of $120 \times 12 \mathrm{~m}^{2}$ as shown in Figure 2a. Transmitter node was located in the border of the parking as shown in Figure $2 b$, where Figure $2 c$ show the movable receiver node which contains ZigBee and laptop supported by data acquisition software to record the RSSI values. The Receiver node moved on a regular path every $5 \mathrm{~m}$ and received the beacons from the transmitter node. A total of 2,300 samples are collected from 23 locations, a hundred samples are collected for each location.

Same transmitter and receiver are used in the indoor experiment. The indoor experiment is achieved in the corridor of the LABs building in the Electrical Engineering Technical College as shown in Figure 3a. Because of area limitations, the total number of points was decreased to 16 points. A total of 1,600 samples are collected from 16 locations, a hundred samples are collected for each location. The measurements are performed in the normal working hours students in LABs to take into account the effect of the signal propagation. The corridor had an area of $33 \times 6 \mathrm{~m}^{2}$ as shown in Figure $3 \mathrm{~b}$. The maximum distances between the transmitter and the receiver node were $30 \mathrm{~m}$.

(A)

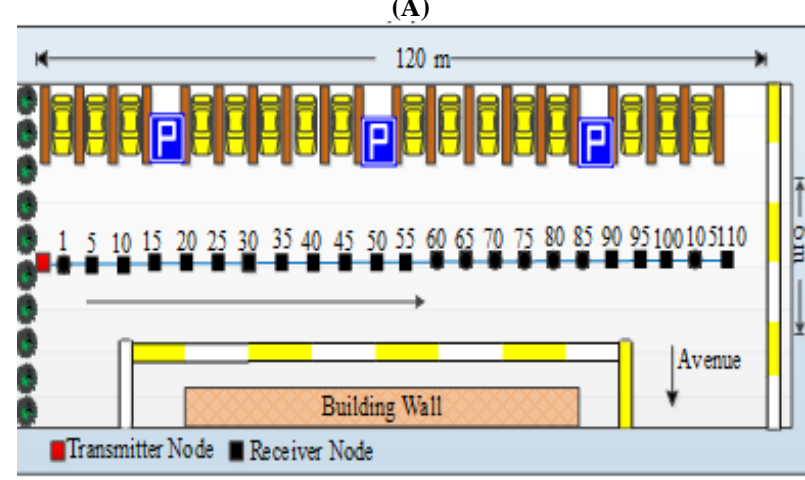

(B)

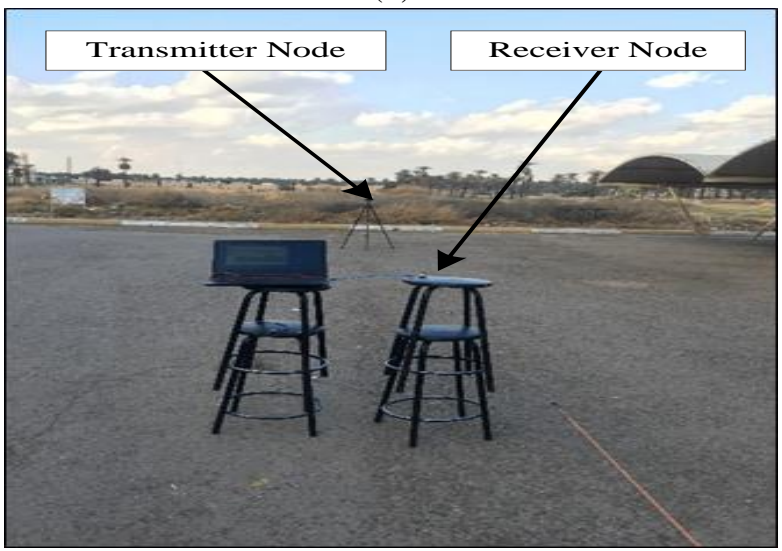

(C)

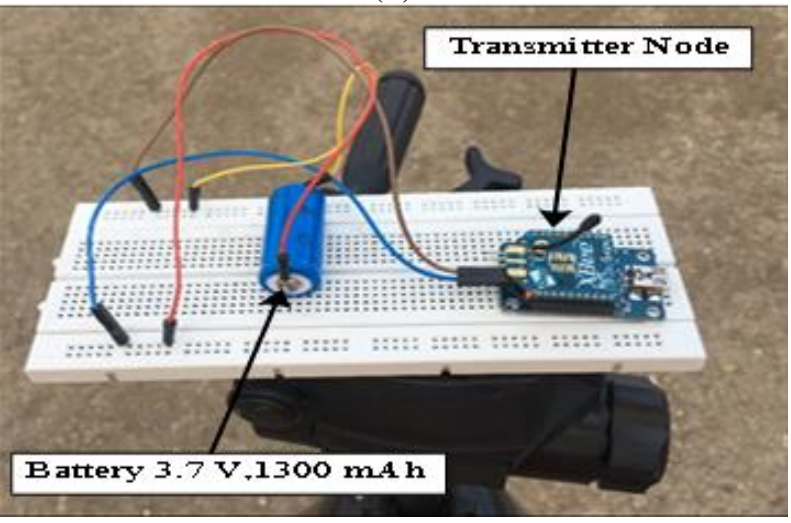

Fig. 2: Configuration of Outdoor Experiment (A) Tested Area, (B) Photo of Transmitter and Receiver Nodes, and (C) Photo of Transmitter Node.

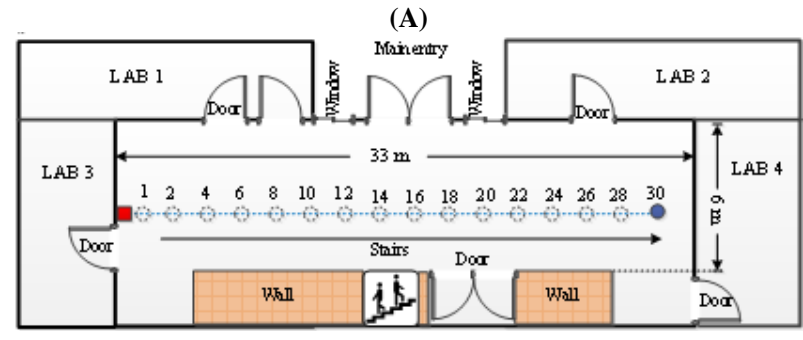

(B)

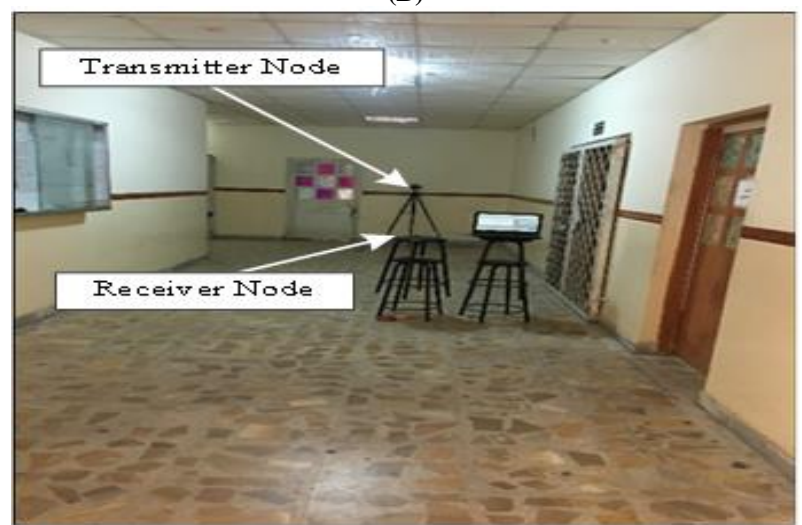

Fig. 3: Configuration of Indoor Experiment (A) Tested Area, (B) Photo of Transmitter and Receiver Nodes Inside Tested Area.

\section{RSSI measurements}

In the outdoor environment, 23 predefined positions are measured RSSI values. At each position, One- hundred samples were recorded, as a one sample per second. Each sample comprises one data packet; each data packet includes 20 bytes. The One- hundred RSSI values were averaged for each location. Figure 4 shows the measured RSSI in outdoor and indoor environments.

In the indoor environment, 16 predefined positions are measured RSSI values. At each position, One- hundred samples were recorded, as a one sample per second. Each sample contains one data packet; each data packet involves 20 bytes. The RSSI values are averaged for each location. All received RSSI for outdoor and indoor are greater than the receiver sensitivity as shown in Figure 4. Therefore, the data packets delivery may be received without packets losses.

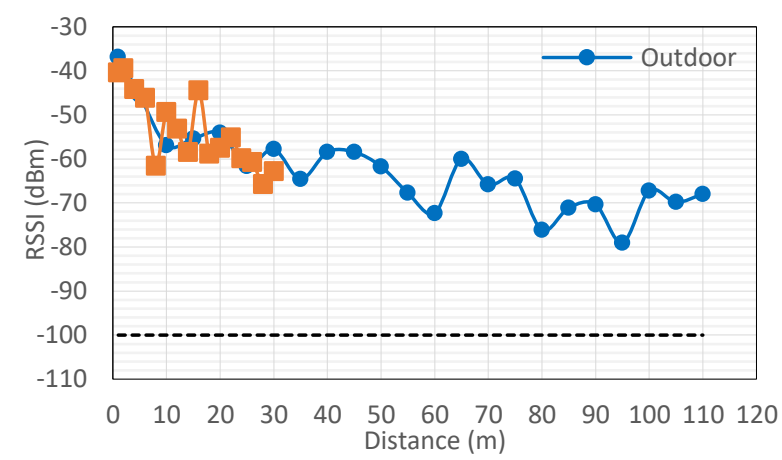

Fig. 4: RSSI Measurements with Respect to Distance in Outdoor and indoor Environments.

\section{Results evaluation}

\subsection{Path loss modelling}

The LNSM parameters were estimated based on the measurements of the averaged RSSI for predefined outdoor and indoor locations. To get the LNSM and related parameters, the relationship between the measured averaged RSSI and the logarithmic scale of the predefined positions is plotted for each outdoors and indoors, as shown 
in Figures 5 and 6 , respectively. The path loss exponents $\beta$ and standard deviation $\mathrm{x}$ can be gotten by using the linear fit line over the curves in both figures. Consequently, the estimated regression line for each outdoors and indoors can be formed by the following equations 4 and 5 , respectively.

$$
\begin{aligned}
& y_{1}=-17.435 x_{1}-35.149 \text { (outdoor) } \\
& y_{2}=-16.168 x_{2}-36.768 \text { (indoor) }
\end{aligned}
$$

Where $y$ denotes the mean of RSSI in $\mathrm{dBm}$, $\mathrm{x} 1$, and $\mathrm{x}_{2}$ is the logarithmic scale of the distance $d$ in meters.

Subsequently, Equations 4 and 5 will become

$$
\begin{aligned}
& \operatorname{RSSI}(\mathrm{dBm})=-17.435 \log \left(\mathrm{l} / \mathrm{l}_{\mathrm{o}}\right)-35.149 \\
& \operatorname{RSSI}(\mathrm{dBm})=-16.168 \log \left(\mathrm{l} / \mathrm{l}_{\mathrm{o}}\right)-36.768
\end{aligned}
$$

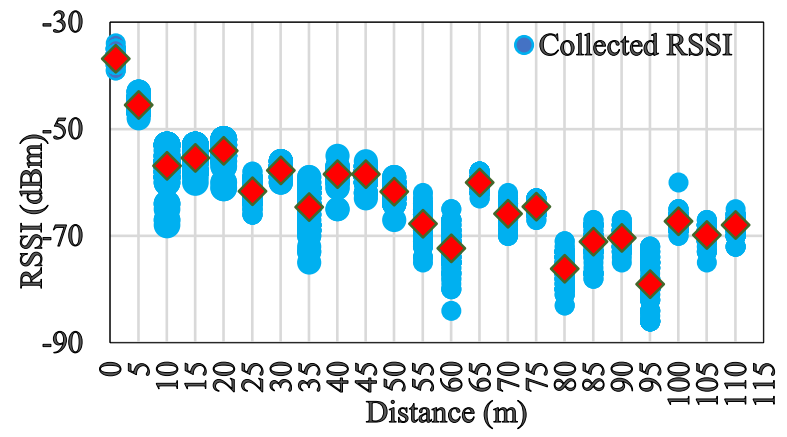

Comparing Equations 6 and 7 with Equation 3. Equation 8 and 9 for outdoor and indoor, respectively can be used to compute the path loss exponent and standard deviation.

$$
\begin{aligned}
& 10 \beta=17.435 \text { and } W_{t}-W_{o}+\sigma=-35.149 \\
& 10 \beta=16.168 \text { and } W_{t}-W_{o}+\sigma=-36.768
\end{aligned}
$$

Which $W_{t}$ is $5 \mathrm{dBm}$ for indoor and outdoor and $W_{o}$ is $36.85 \mathrm{dBm}$ for outdoor and 40.33 for indoor (obtained from real measurement at $l_{o}=1 \mathrm{~m}$ ). Then, the parameters of the LNSM can be computed as shown in Table 1 .

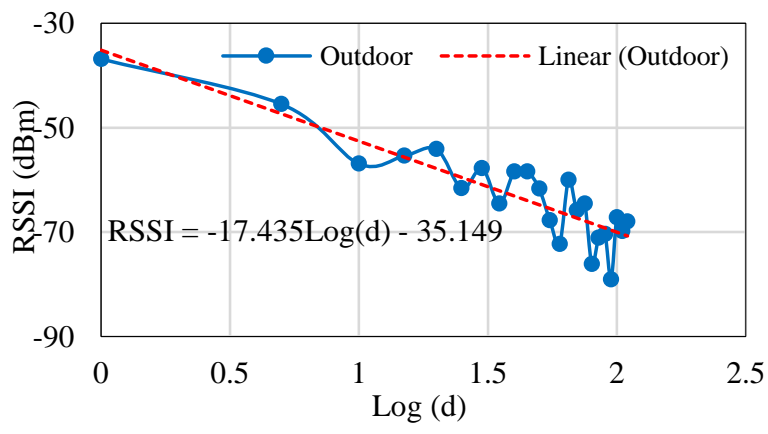

Fig. 5: The Fitting Curve for Outdoor Environments.

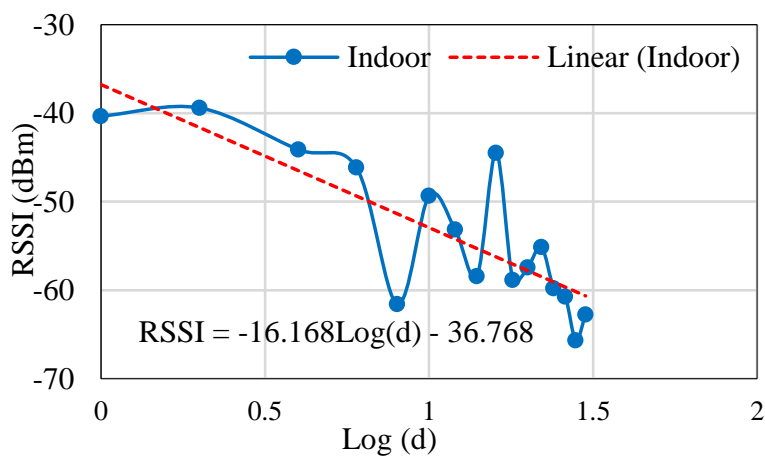

Fig. 6:.The Fitting Curve for Indoor Environments.

\subsection{Variances of RSSI}

Because of one hundred RSSI samples were recorded at each 23 positions in outdoor environment, the variances of RSSI values is expected due to signal path-loss, multipath propagation and shadowing. Figure 7 shows the measured RSSI are fluctuated at each position as illustrated in blue circles and the averaged RSSI as a red diamond points. The figure shows that the RSSI values have low fluctuation in some locations (e.g., 1, 5, 25, 30, 65, $75 \mathrm{~m}$ ) and high fluctuation in another positions (e.g., 10, 35, 55, 60, 80-95 m). Figure 8 shows the fluctuations at each position as demonstrated in blue circles and the averaged RSSI as a red diamond points. The figure illustrates that the RSSI values have low fluctuation in some locations (e.g., 2, 6, 10, 16, 20, $22 \mathrm{~m}$ ) and high fluctuation in an-

\begin{tabular}{|c|c|c|c|c|c|}
\hline \multirow[t]{2}{*}{ Parameters } & \multirow[t]{2}{*}{$\begin{array}{l}\text { Sym- } \\
\text { bol }\end{array}$} & \multirow[t]{2}{*}{ Unit } & \multicolumn{2}{|c|}{$\begin{array}{l}\text { Measured val- } \\
\text { ues }\end{array}$} & \multirow[t]{2}{*}{$\begin{array}{l}\text { Theoretical val- } \\
\text { ues for outdoor } \\
\text { and indoor }\end{array}$} \\
\hline & & & $\begin{array}{l}\text { Out- } \\
\text { door }\end{array}$ & $\begin{array}{l}\text { In- } \\
\text { door }\end{array}$ & \\
\hline $\begin{array}{l}\text { Reference } \\
\text { distance }\end{array}$ & $l_{o}$ & $\mathrm{~m}$ & 1 & 1 & $\begin{array}{l}0.1-10 \\
(1 \mathrm{~m} \text { is used })\end{array}$ \\
\hline $\begin{array}{l}\text { Path loss at } \\
\text { a distance } \\
d_{o}\end{array}$ & $\begin{array}{l}\mathrm{WL} \\
\left(l_{o}\right)\end{array}$ & $\mathrm{dBm}$ & -36.85 & $-\overline{40.33}$ & $\begin{array}{l}37 \\
\text { (from Friis equa- } \\
\text { tion) }\end{array}$ \\
\hline $\begin{array}{l}\text { Path loss ex- } \\
\text { ponent }\end{array}$ & $\beta$ & -- & 1.74 & 1.62 & $\begin{array}{l}2 \text { outdoor [ } 34, \\
35] \\
1.6-1.8 \text { indoor } \\
\text { ( } 1.8 \text { is used for } \\
\text { indoor) }\end{array}$ \\
\hline $\begin{array}{l}\text { Standard de- } \\
\text { viation }\end{array}$ & $\sigma$ & $\mathrm{dB}$ & 3.299 & 1.438 & $\begin{array}{l}2-14 \text { [36] } \\
2 \text { is used for out- } \\
\text { door and indoor }\end{array}$ \\
\hline
\end{tabular}
other positions (e.g., 1, 4, 8, 12, 14, 18, 22-32 m). Obviously, more fluctuations are occur in the indoor environments relative to outdoor due to the reflected signal from roof, wall, windows and doors which causes multi-path effect.

Table 1: The Parameters of the LNSM

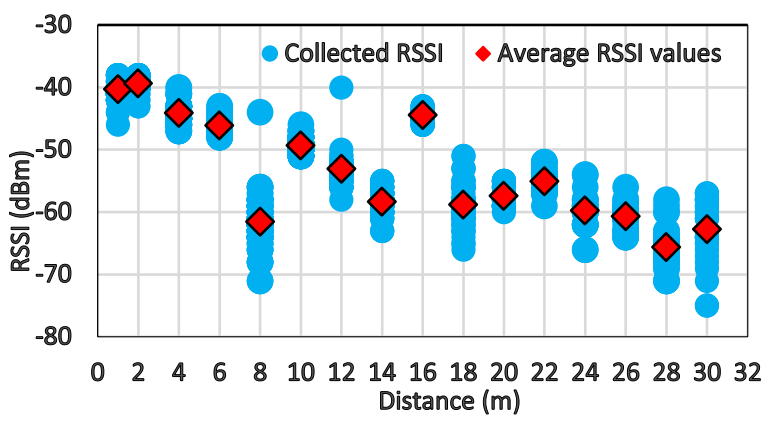

Fig. 7: Variances of RSSI Values with Respect to the Distance for Outdoor

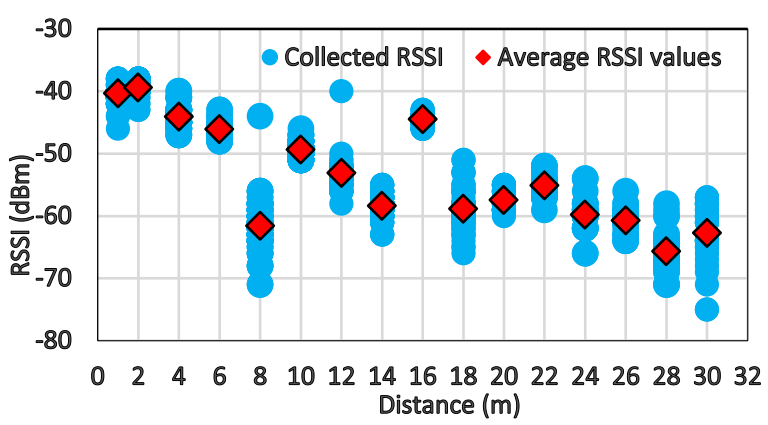

Fig. 8: Variances of RSSI Values with Respect to the Distance for Indoor.

\subsection{Packets losses}

Figure 9 shows the data packets percentage received by the receiver node with different distances for the outdoor and indoor environments. For outdoor, all the received packets are $100 \%$, whereas it is $95 \%$ on $10 \mathrm{~m}$ because there are some cars and students are moved in the tested area during real experiment which it makes attenuation 
in the signal. In addition, the path loss increases with distance. For indoor, all the received packets are $100 \%$, whereas it is $96.67 \%$ on $2 \mathrm{~m}$ because students movement in the tested area which it makes more reflection from ground, roof, and walls in the signal. In the all locations, the received data packets in indoor is better than outdoor because of aforementioned reason above in indoor environments. The Packet loss can be compared with previous work such as [32] which used the ZigBee wireless protocol to evaluated the adopted WSN for outdoor and indoor environments as shown in Figure 9. The figure shows the percentage of data packets received by the movable node for both indoor and outdoor surroundings versus distance between the movable node and anchor node (located at the center of track cycling application) [32].

The figure disclosed that our measurements outperformed that previous work for both indoor and outdoor. The received data packets of the ref. [32] is varied between $88 \%-96 \%$ (indoor) and $80 \%-100 \%$ (outdoor), whereas for the current work have $100 \%$ for all distances value except $95 \%$ at $10 \mathrm{~m}$ (outdoor) and $96.67 \%$ at $2 \mathrm{~m}$ (indoor). However, the data packets received is $100 \%$ at high distance of 110 $\mathrm{m}$ for outdoor. This superiority for current work is attributed to the fact that the using of XBeeS2C which have high communication distance with low power consumption than XBee S2 (which used in [32]).

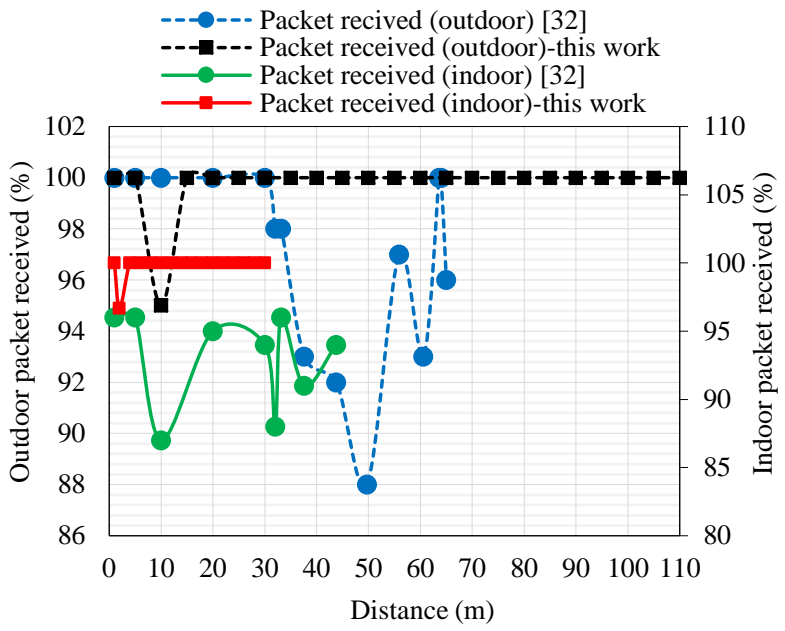

Fig. 9: Received Data Packet Against Distance for Indoor and Outdoor Environments.

\subsection{Standard deviation}

The examined standard deviation of RSSI measurement for outdoor environment with taking into consideration to the distance of each location is shown in Figure 10. The figure demonstrates that the RSSI values are more diverged in location 10 and $35 \mathrm{~m}$ due to the same reason mentioned for outdoor environment. In addition, the RSSI values are more deviated at 60 and $95 \mathrm{~m}$ because the far distance when the transmitter node go away from receiver node.

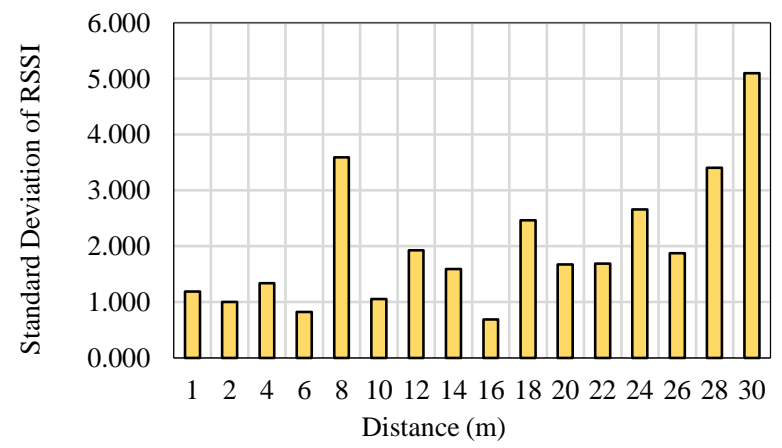

Fig. 10: The Standard Deviation of the RSSI Values with Respect to the Distance for Outdoor Readings.

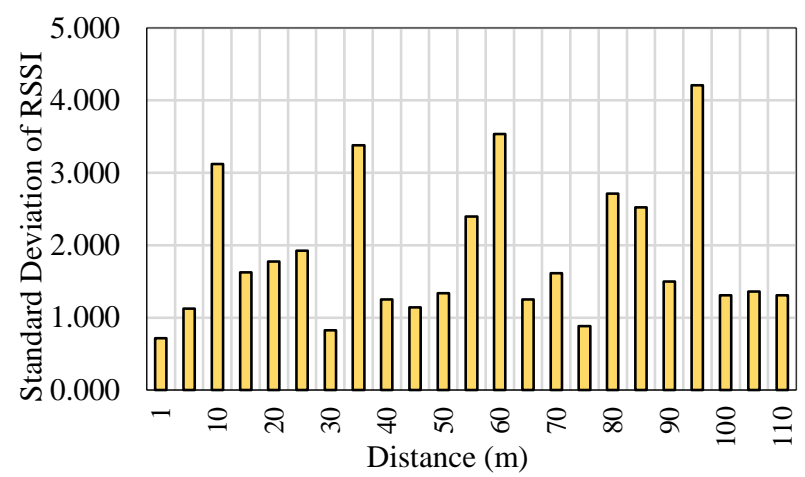

Fig. 11: The Standard Deviation of the RSSI Values With Respect to the Distance for Indoor Readings.

The investigated standard deviation of RSSI measurement for indoor environment with taking into consideration to the distance of each location as shown in Figure 11. The figure illustrates that the RSSI values are more diverged with distance increasing due to the same reason mentioned for indoor environment.

\section{Conclusion}

The main objective of this paper is to measure the path loss model with its parameters between transmitter node and receiver node using ZigBee wireless protocol in outdoor and indoor environments. The path loss model is derived based on LNSM by linear fit relationship between the RSSI values in each position and the distance. In addition, the standard deviation and the path loss exponent were also measured. Moreover, the variances, standard deviations, and packets loss of RSSI have been investigated. This study was used XBee S2 wireless technology because it can measure RSSI without addition hardware, cheap, easy, efficient power consumption, and long communication coverage area. This study disclosed that the current work overcome the previous work in terms of data packet received. The current work can be extended in future by using an artificial intelligent techniques (such as neural network) or optimization algorithm (such as Particle Swarm Optimization) for accurate distance estimation between transmitter and receiver.

\section{Acknowledgement}

The authors would like to thank the staff in Department of Medical Techniques Engineering, College of Electrical Engineering Techniques, and Middle Technical University (MTU) for their support to conduct this work.

\section{References}

[1] Bhuvaneswari P, Vaidehi V, Saranya MA. Distance based transmission power control scheme for indoor wireless sensor network. Transactions on computational science XI: Springer; 2010. p. $207-$ 22.

[2] Pinto S, Cabral J, Gomes T. We-care: An IoT-based health care system for elderly people. Industrial Technology (ICIT), 2017 IEEE International Conference on: IEEE; 2017. p. 1378-83.

[3] Gharghan SK, Nordin R, Ismail M. Development and validation of a track bicycle instrument for torque measurement using the zigbee wireless sensor network. International Journal on Smart Sensing and Intelligent Systems.2017 10:124-45. https://doi.org/10.21307/ijssis2017-206.

[4] Jawad HM, Nordin R, Gharghan SK, Jawad AM, Ismail M. EnergyEfficient wireless sensor networks for precision agriculture: A review. Sensors. 201717 1781. https://doi.org/10.3390/s17081781.

[5] Gharghan SK, Nordin R, Ismail M. An ultra-low power wireless sensor network for bicycle torque performance measurements. Sensors. 201515 11741-68. https://doi.org/10.3390/s150511741.

[6] Gharghan SK, Nordin R, Ismail M. Energy-efficient ZigBee-based wireless sensor network for track bicycle performance monitoring. Sensors. 201414 15573-92. https://doi.org/10.3390/s140815573. 
[7] Di Francesco M, Anastasi G, Conti M, Das SK, Neri V. Reliability and energy-efficiency in IEEE 802.15. 4/ZigBee sensor networks: an adaptive and cross-layer approach. IEEE Journal on selected areas in communications. 2011 https://doi.org/10.1109/JSAC.2011.110902.

[8] Hebel M, Bricker G. Getting started with XBee RF modules. Tutorial Parallax Inc Consulta. 20108.

[9] Bell C. Beginning sensor networks with Arduino and Raspberry Pi: Apress; 2014.

[10] Inc. DI. XBee®/XBee-PRO S2C Zigbee, Available: https://www.digi.com/resources/documentation/digidocs/pdfs/90002002.pdf (accessed on 21 March 2018).

[11] MaxStream, Inc. - a Digi International brand, XBee ${ }^{\mathrm{TM}}$ Series 2 OEM RF Modules. Available: http://www.farnell.com/datasheets/27606.pdf (accessed on 21 March 2018).

[12] Digi International Inc. DIGI XBEE® S1 802.15.4 RF MODULES, Available: https://www.digi.com/pdf/ds_xbeemultipointmodules.pdf (accessed on 21 March 2018).

[13] Poutanen J, Haneda K, Salmi J, Kolmonen V-M, Koivunen J, Almers $\mathrm{P}$, et al. Analysis of radio wave propagation from an indoor hall to a corridor. Antennas and Propagation Society International Symposium, 2009 APSURSI'09 IEEE: IEEE; 2009. p. 1-4 https://doi.org/10.1109/APS.2009.5172169.

[14] Noori N, Karimzadeh-Baee R, Abolghasemi A. An empirical ultra wideband channel model for indoor laboratory environments. Radioengineering. 200918 68-74.

[15] Geng S, Vainikainen P. Millimeter-wave propagation in indoor corridors. IEEE Antennas and Wireless Propagation Letters. 20098 1242-5. https://doi.org/10.1109/LAWP.2009.2035723.

[16] Lim SY, Yun Z, Baker JM, Celik N, Youn H-s, Iskander MF. Propagation modeling and measurement for a multifloor stairwell. IEEE Antennas and Wireless Propagation Letters. 20098 583-6. https://doi.org/10.1109/LAWP.2009.2021870.

[17] Valcarce A, Zhang J. Empirical indoor-to-outdoor propagation model for residential areas at $0.9-3.5 \mathrm{GHz}$. IEEE Antennas and Wireless Propagation Letters. $2010 \quad 9 \quad 682-5$. https://doi.org/10.1109/LAWP.2010.2058085.

[18] Benkic K, Malajner M, Planinsic P, Cucej Z. Using RSSI value for distance estimation in wireless sensor networks based on ZigBee. Systems, signals and image processing, 2008 IWSSIP 2008 15th international conference on: IEEE; 2008. p. 303-6. https://doi.org/10.1109/IWSSIP.2008.4604427.

[19] Malajner M, Benkic K, Planinsic P, Cucej Z. The accuracy of propagation models for distance measurement between WSN nodes. Systems, Signals and Image Processing, 2009 IWSSIP 2009 16th International Conference on: IEEE; 2009. p. 1-4 https://doi.org/10.1109/IWSSIP.2009.5367782.

[20] Al Alawi R. RSSI based location estimation in wireless sensors networks. Networks (ICON), 2011 17th IEEE International Conference on: IEEE; 2011. p. 118-22.

[21] Dezfouli B, Radi M, Razak SA, Hwee-Pink T, Bakar KA. Modeling low-power wireless communications. Journal of Network and Computer Applications. 201551: 102-26. https://doi.org/10.1016/j.jnca.2014.02.009.

[22] Adewumi O, Djouani K, Kurien A. Performance evaluation of RSSI based distance measurement for localization in wireless sensor networks. International Conference on e-Infrastructure and e-Services for Developing Countries: Springer; 2012. p. 74-83.

[23] Miramontes R, Aquino R, and Flores a, Rodríguez G, Anguiano R, Ríos A, et al. PlaIMoS: a remote mobile healthcare platform to monitor cardiovascular and respiratory variables. Sensors. 201717176. https://doi.org/10.3390/s17010176.

[24] Prakash R, Ganesh AB, Girish SV. Cooperative wireless network control based health and activity monitoring system. Journal of medical systems. 2016 40:216. https://doi.org/10.1007/s10916-0160576-4.

[25] Watthanawisuth N, Lomas T, Wisitsoraat A, Tuantranont A. Wireless wearable pulse oximeter for health monitoring using ZigBee wireless sensor network. Electrical Engineering/Electronics Computer Telecommunications and Information Technology (ECTICON), 2010 International Conference on: IEEE; 2010. p. 575-9.

[26] Wong A, McDonagh D, Omeni O, Nunn C, Hernandez-Silveira M, Burdett A. Sensium: An ultra-low-power wireless body sensor network platform: Design \& application challenges. Engineering in Medicine and Biology Society, 2009 EMBC 2009 Annual International Conference of the IEEE: IEEE; 2009. p. 6576-9.

[27] Chen S-K, Kao T, Chan C-T, Huang C-N, Chiang C-Y, Lai C-Y, et al. A reliable transmission protocol for zigbee-based wireless patient monitoring. IEEE Transactions on Information Technology in Biomedicine. 2012

16:6-16. https://doi.org/10.1109/TITB.2011.2171704.

[28] Liu F, Zhu H, Gu Z, Liu Y. A linear localization algorithm for wireless sensor network based on RSSI. Advanced Research on Computer Education, Simulation and Modeling: Springer 2011. p. 384-9.

[29] Tang L, Liu M, Wang K-C, Huang Y, Yang F, and Zhang D. Study of path loss and data transmission error of IEEE 802.15.4 compliant wireless sensors in small-scale manufacturing environments. The International Journal of Advanced Manufacturing Technology. 2012 63 659-69. https://doi.org/10.1007/s00170-012-3928-3.

[30] Pivato P, Palopoli L, Petri D. Accuracy of RSS-based centroid localization algorithms in an indoor environment. IEEE Transactions on Instrumentation and Measurement. $201160 \quad 3451-60$. https://doi.org/10.1109/TIM.2011.2134890.

[31] Chuang P-J, Jiang Y-J. Effective neural network-based node localisation scheme for wireless sensor networks. IET Wireless Sensor Systems.2014 4:97-103.

[32] Gharghan SK, Nordin R, Ismail M. Energy efficiency of ultra-lowpower bicycle wireless sensor networks based on a combination of power reduction techniques. Journal of Sensors. 20162016.

[33] Lian K-Y, Hsiao S-J, Sung W-T. Intelligent multi-sensor control system based on innovative technology integration via ZigBee and $\mathrm{Wi}$ Fi networks. Journal of network and computer applications. 201336 756-67. https://doi.org/10.1016/j.jnca.2012.12.012.

[34] Ta X, Mao G, Anderson BD. On the giant component of wireless multihop networks in the presence of shadowing. IEEE Transactions on Vehicular Technology. 2009; 58:5152-63. https://doi.org/10.1109/TVT.2009.2026480.

[35] Noh S-K, Kim K-S, Ji Y-K. Design of a room monitoring system for wireless sensor networks. International Journal of Distributed Sensor Networks. 2013 9:189840. https://doi.org/10.1155/2013/189840.

[36] Sahu PK, Wu EH-K, Sahoo J. DuRT: Dual RSSI trend based localization for wireless sensor networks. IEEE Sensors Journal. 2013; 13:3115-23. https://doi.org/10.1109/JSEN.2013.2257731. 